\title{
Evaporation and boiling crisis of droplets alcohol solution
}

\author{
S.Y. Misyura ${ }^{1, *}$, V.S. Morozov ${ }^{1}$ \\ ${ }^{1}$ Institute of Thermophysics of SBRA, 630090 Novosibirsk, Russia
}

\begin{abstract}
Evaporation and boiling crisis of droplets ethanol aqueous solution were studied experimentally. The evaporation intensity depends on the nucleate boiling, solution diffusion, a change in physical characteristics with time and droplet interfacial surface area. At nucleate boiling in a droplet, most evaporation relates to a growth in the droplet surface area and only $20 \%$ relates to the diffusion effect and a variation in the thermophysical coefficients. At boiling crisis, experimental dependence for vapor layer height on overheating was observed. At Leidenfrost temperature, the height of the vapor layer was many times higher than the surface microroughness value of the wall. There are oscillates of liquid-vapor interface, and this increases the transitional temperature range associated with a boiling crisis of droplets.
\end{abstract}

\section{Introduction}

The evaporation of solutions is widely applied in microelectronics, energy apparatuses and chemical technologies. There is boiling crisis at high heat fluxes. The formed vapor layer during the crisis reduces the heat transfer coefficient by several orders, resulting the wall overheating and wall destruction. The lowest temperature boundary of this regime with stable vapor layer (without touching the wall and the liquid) is referred to as the Leidenfrost point (LFP). Predictions for LFP can be obtained on the basis of understanding of boiling key mechanisms. Condensation and evaporation of pure liquids differ significantly from absorption and desorption of salt solutions or phase transition of alcohol solutions [1-9]. At desorption of droplet solution there are salt spot and crystals, which depend on the wettability (droplet contact angle). Influence of the contact angle, wettability and surface wall properties on the droplet evaporation is presented in the work [10]. Vapor film formation and boiling crisis in mini channels lead to flow locking and apparatus breakages [11]. Acceleration of the flow affects the transport mechanisms in the gas mixture [12].

Phase transitions rates (evaporation, crystallization, dissociation) strongly depend on both the external heat flux and internal local heat exchanges [13-19] and on impurities [20-21]. The measurement procedure in non-isothermal flow is presented in [22].

\footnotetext{
Corresponding author: misura@itp.nsc.ru
} 


\section{Experimental data}

Evaporation curves is shown in Fig. 1. The presentation of the experimental data in Fig. 1(a) is not suitable because there are several factors affecting evaporation: the interface area; diffusivity, evaporation heat, heat transfer coefficient. The droplet wetting surface for alcohol and pure water differ by several times of magnitude. The curves in Fig. 1(b) are given taking into account the interface area. Thus at nucleate boiling in a droplet, the largest part of the evaporation relates to a growth in the droplet surface area and only $20 \%$ relates to the diffusion effect (formation of a high transverse concentration gradient) and a variation in the thermophysical coefficients.

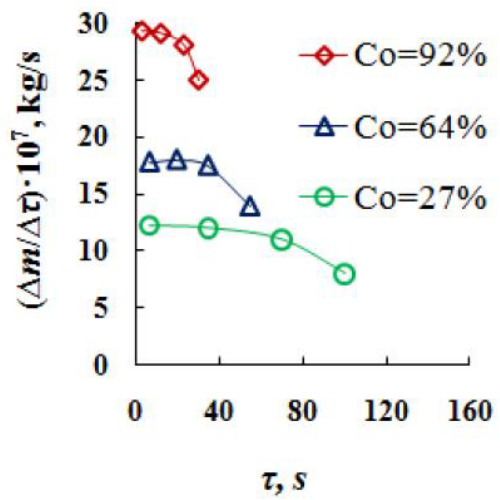

(a)

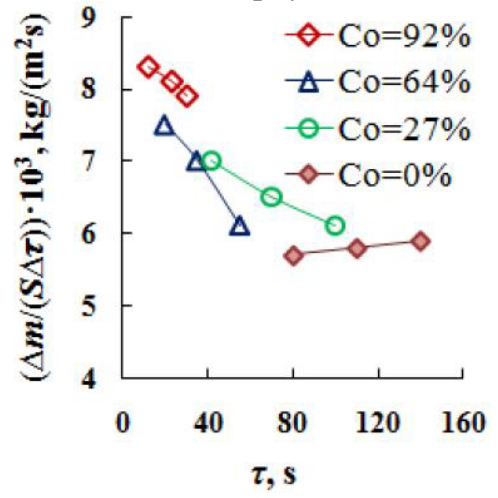

(b)

Fig. 1. a) Changing in the droplet mass without taking into account the evaporation area; b) Evaporation droplet rate considering the interface area $\left(C_{0}\right.$ - mass alcohol concentration).

The vapor layer height (at the boiling crisis) $\delta_{v}$ increases with wall overheating $\Delta T_{w}$ and repeatedly exceeds the height of the wall surface microroughness (Fig. 2). Repeatedly increased thermal images and high speed shooting indicate the presence of long-wave oscillations on the droplet interphase surface.

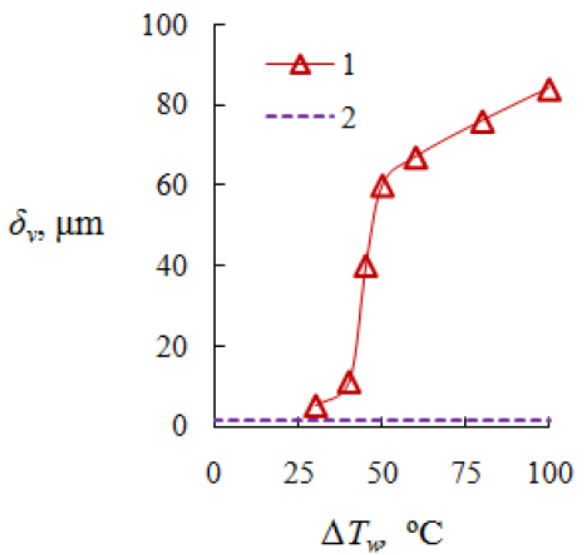

Fig. 2.1 - Vapor layer thickness $\delta_{v}$ vs. overheating $\Delta T_{w}, 2$ - maximal microroughness height of the wall surface.

This work was supported by the Russian Science Foundation (grant N 15-19-10025). 


\section{References:}

1. S.Ya. Misyura, Int. J. Therm. Sci. 92, (2015).

2. G.V. Kuznetsov, D.V. Feoktistov, E.G. Orlova, Thermophys. Aeromech. 23 (2016)

3. G.V. Kuznetsov, D.V. Feoktistov, E.G. Orlova, J. Eng. Phys. Thermophys. 89 (2016)

4. V.E. Nakoryakov, S.Y. Misyura, S.L. Elistratov, J. Eng. Thermophys. 22 (1), (2013)

5. V.E. Nakoryakov, S.Y. Misyura, S.L. Elistratov, J. Eng. Thermophys.20 (4), (2011).

6. V.E. Nakoryakov, S.Y. Misyura, S.L. Elistratov, Thermal Science, 16 (4) (2012)

7. V.E. Nakoryakov, S.Y. Misyura, Doklady Physics, 59 (10) (2014)

8. S.Ya. Misyura, Int. J. Heat Mass Transfer, 71 (2014)

9. S.Y.Misyura, Appl. Therm. Eng. 113 (25), (2017)

10. J. Wang, Scientific Reports, 5, 8474 (2015)

11. V.E. Nakoryakov, S.Y. Misyura, S.L. Elistratov, R.A. Dekhtyar, J. Eng. Thermophys. 23 (4), (2014)

12. V.P. Lebedev, V.V. Lemanov, S.Y. Misyura, V.I. Terekhov, Fluid dynamics, 28 (1993)

13. A.K. Rozentsvaig, Ch.S. Strashinskii, Int. J. Heat Mass Transfer, 102, 555 (2016)

14. A. Pavlenko, V. Zhukov, N. Pecherkin, V. Chekhovich, O. Volodin, A. Shilkin, C. Grossmann, AIChE J. 60 (2), (2014)

15. S.Y.Misyura, Scientific Reports, 6, 30324 (2016)

16. A.A. Chernov, Int. J. Heat Mass Transfer, 52 (2009)

17. V.E.Nakoryakov, S.Y.Misyura, S.L. Elistratov, J. Eng. Thermophys. 22 (3), (2013)

18. S.Y. Misyura, Energy, 103 (2016)

19. V.E. Nakoryakov, S.Y. Misyura, Energy Fuels, 27, (2013)

20. R.S. Volkov, G.V. Kuznetsov, P.A. Strizhak, Int. J. Heat Mass Transfer, 96 (2016)

21. R. Iegorov, T. Valliulin, P. Strizhak, Appl. Therm. Eng. 106 (2016).

22. S.L. Elistratov, O.V. Vitovskii, E.Yu. Slesareva, J. Eng. Thermophys. 24 (1), (2015) 